\title{
Contribution of ENPP1, TCF7L2, and FTO polymorphisms to type 2 diabetes in mixed ancestry ethnic population of South Africa
}

\author{
Yandiswa Y. Yako ${ }^{1}$, Jabulisile H. Madubedube ${ }^{2}$, Andre P. Kengne ${ }^{3,4}$, Rajiv T. Erasmus ${ }^{5}$, \\ Tahir S Pillay ${ }^{6}$, Tandi E. Matsha ${ }^{2}$.
}

1. Department of Surgery, Faculty of Health Sciences, University of Witwatersrand, South Africa.

2. Department of Biomedical Sciences Faculty of Health and Wellness Sciences, Cape Peninsula University of Technology, Cape Town, South Africa.

3. Non-Communicable Diseases Research Unit, South African Medical Research Council, Cape Town, South Africa.

4. Department of Medicine, University of Cape Town, Cape Town, South Africa.

5. Division of Chemical Pathology, Faculty of Medicine and Health Sciences, National Health Laboratory Service (NHLS) and University of Stellenbosch, Cape Town, South Africa.

6. Institute for Cellular and Molecular Medicine, Molecular Endocrinology, University of Pretoria

\begin{abstract}
Background: Transcription factor 7-like 2 gene (TCF7L2), fat mass and obesity-associated gene (FTO), and ectonucleotide pyrophosphatase/phosphodiesterase gene (ENPP1) are known risk loci for type 2 diabetes (T2DM) mostly in European populations.

Objectives: To assess the association of these genes with T2DM risk in a South African mixed-ancestry population.

Methods: Five hundred and sixty six participants were genotyped for ENPP1-rs997509 and -rs1044498, FTO-9941349 and -rs3751812, TCF7L2-rs12255372 and -rs7903146 polymorphisms using Taqman genotyping assays and validated by automated sequencing to assess the association of the polymorphisms with cardiometabolic traits.

Results: In logistic regression models adjusted for age, sex, body mass index (BMI) and insulin resistance, minor allele of rs997509 was associated with a higher risk of prevalent T2DM under a recessive model [odd ratio 4.60 (95\% confidence interval: 1.07 to 19.86); $\mathrm{p}=0.040]$.Under additive model, the rs7903146 [1.43 (1.00 to 2.04); $\mathrm{p}=0.053$ ] and rs9941349 [1.43 (1.00 to 2.04); $\mathrm{p}=0.052]$ minor alleles showed marginally significant associations with a high risk of T2DM. However, only the rs7903146 alleles $(\mathrm{p}=0.011)$ and genotypes $(\mathrm{p}=0.025)$ distributions were statistically significantly different between diabetic and non-diabetic individuals.

Conclusion: Our findings demonstrate that ENPP1, TCF7L2, and FTO may predispose to T2DM in the mixed-ancestry population.

Keywords: Type 2 diabetes, genetics, ENPP1, TCF7L2, FTO, Africa

DOI: http://dx.doi.org/10.4314/ahs.v15i4.14

Cite as: Yako YY, Madubedube JH, Kengne AP, Erasmus RT, Pillay TS, Matsha TE. Contribution of ENPP1, TCF7L2, and FTO polymorphisms to type 2 diabetes in mixed ancestry ethnic population of South Africa. Afri Health Sci. 2015;15(4):1149-60. http:// dx.doi. org/10.4314/abs.v15i4.14
\end{abstract}

\author{
Corresponding author: \\ Tandi E. Matsha, \\ Biomedical Sciences, \\ Faculty of Health and Wellness Sciences, \\ Cape Peninsula University of Technology, \\ P.O. Box 1906, Bellville 7530, \\ Cape Town, South Africa \\ Tel: +27 21959 6366, Fax: +27 219596760 \\ Email:matshat@cput.ac.za
}

\section{Introduction}

The completion of the human genome a decade ago has paved a way for a better understanding of the genetic basis of common and complex diseases. Through candidate gene and linkage analyses, and recently genome wide association studies (GWAS), several single nucleotide polymorphisms (SNPs) have been reported as risk loci for type 2 diabetes in European (39 loci) and Asian (19 loci) population groups ${ }^{1,2}$. The majority of these loci seem to influence beta-cell function and insulin sensitivity, although the mechanisms by which 
they impair such functions are yet to be determined. Among these loci are transcription factor 7-like 2 gene (TCF7L2), fat mass and obesity-associated gene (FTO), and ectonucleotide pyrophosphatase/phosphodiesterase gene (ENPP1).

Transcription factor 7-like 2 gene is the first T2DM risk locus to be identified through large-scale association analyses in European populations ${ }^{3}$. The protein encoded by the TCF7L2 is a transcription factor of the Wnt signalling pathway, which plays a crucial role in beta-cell function, insulin secretion, and proglucagon gene expression $^{4,5}$. The first surge of T2DM GWA identified among others the fat mass and obesity-associated gene (FTO), which is the strongest obesity genetic risk factor reported so far ${ }^{6,7}$. It has been shown to confer susceptibility to T2DM in the presence ${ }^{8,9}$ and absence of obesity $^{10,11}$. The most widely studied SNP of the ENPP1, K121Q (rs1044498), has produced conflicting results in relation to T2DM susceptibility ${ }^{12-14}$. The ENPP1-encoded protein (also known as plasma cell-1, PC-1) is a class II transmembrane glycoprotein that interacts with insulin receptor and inhibits subsequent insulin-signalling through its impaired beta-subunit autophosphorylation, thus causing insulin resistance ${ }^{15}$.

Although few number of T2DM susceptibility genes discovered through candidate gene, linkage analyses, and GWA studies elsewhere have been replicated in African studies ${ }^{16-21}$, it must also be emphasized that many other association studies failed to show significant and replicable findings $\mathrm{s}^{22-24}$. Therefore in this study we investigated three genes, namely, TCF7L2, FTO, and ENPP1 as risk factors for T2DM in a South African ethnic population group of Mixed-ancestry (Coloureds) with a unique genetic architecture. Structure analysis conducted in this population revealed that its origin is predominantly Khoesan (32-43\%), Bantu-speaking African (20-36\%), European (21-28\%), and a small proportion Asian $(9-11 \%)^{25}$.

\section{Materials and Methods Ethical approval of the study}

The study was approved by the Faculty of Health and Wellness Sciences Ethics Committee of the Cape Peninsula University of Technology (CPUT) NHREC: REC-230408-014, and was conducted according to the code of ethics of the World Medical Association (Declaration of Helsinki). All participants who were recruit- ed for the study voluntarily signed written consent after the procedures had been fully explained in the language of their choice. Permission to conduct the study was granted by relevant authorities such as city and community authorities.

\section{Study design and population}

The present study was cross-sectional by design, involving participants from a mixed-ancestry ethnic population group residing in Bellville South township in Cape Town, South Africa. The population group has lived in Bellville South since late 1950s. A detailed description of the survey and procedures conducted in the study are available elsewhere ${ }^{26,27}$.

\section{Clinical data}

Clinical data was collected in the form of a standardized questionnaire. During this time, physical examination was conducted with data collection on blood pressure according to World Health Organisation (WHO) guidelines $^{28}$ using a semi-automatic digital blood pressure monitor (Rossmax PA, USA) on the right arm in sitting position, and anthropometric measurements; body weight was measured to the nearest $0.1 \mathrm{~kg}$ with a Sunbeam EB710 digital bathroom scale, which was calibrated and standardized using a weight of known mass. Measurements were recorded with each subject wearing light clothing, without shoes and socks. Waist circumference was determined using a non-elastic tape at the level of the narrowest part of the torso, as seen from the anterior view. All anthropometric measurements were performed three times and their average used for analysis. Participants with no history of doctor diagnosed diabetes mellitus underwent a $75 \mathrm{~g}$ oral glucose tolerance test (OGT'T) as recommended by the $\mathrm{WHO}^{29}$.

\section{Biochemical analysis}

Blood samples were collected from participants after an overnight fast. Blood glucose level and glycated haemoglobin (HbA1c) were measured, respectively, by enzymatic hexokinase method and turbidimetric inhibition immunoassay (Cobas 6000, Roche Diagnostics, Germany). Insulin was determined by a microparticle enzyme immunoassay (Axsym, Abbot). High-density lipoprotein cholesterol (HDL-C) and triglycerides (TG) were estimated by enzymatic colorimetric methods (Cobas 6000, Roche Diagnostics). Low-density lipoprotein cholesterol (LDL-C) was calculated using Friedwald's formula. 


\section{Definitions and calculations}

Body mass index (BMI) was calculated as weight per square meter $\left(\mathrm{kg} / \mathrm{m}^{2}\right)$ and waist-hip-ratio (WHR) as waist/hip circumferences $(\mathrm{cm})$. Diabetes was based on a history of doctor-diagnosis, fasting blood glucose concentration $\geq 7.0 \mathrm{mmol} / \mathrm{L}$ (or $126 \mathrm{mg} / \mathrm{dL}$ ) and/or 2-hour post-OGTT plasma glucose $\geq 11.1 \mathrm{mmol} / \mathrm{L}$ (or $200 \mathrm{mg} / \mathrm{dL}$ ). The homeostatic model assessment of insulin resistance (HOMA-IR) was calculated according to the formula: HOMA-IR = [fasting insulin concentration $(\mathrm{mIU} / \mathrm{L}) \mathrm{x}$ fasting plasma glucose $(\mathrm{mmol} \mathrm{L}] / 22.5$; while functional $\beta$-cells (HOMA-B \%) were estimated using the formula: $20 \times$ fasting insulin $(\mu \mathrm{IU} / \mathrm{ml}) /$ fasting glucose $(\mathrm{mmol} / \mathrm{ml})-3.5$. The fasting insulin resistance index (FIRI) was calculated with the formula: [fasting insulin $(\mu \mathrm{U} / \mathrm{ml}) \times$ fasting glucose $(\mathrm{mM})] / 25$ and the quantitative insulin-sensitivity check index (QUICKI) as: $1 /[\log$ (fasting insulin $(\mu \mathrm{U} / \mathrm{ml}) \times \log$ (fasting glucose $(\mathrm{mg} / \mathrm{dl})]$.

\section{Genotyping}

Genomic DNA was extracted from peripheral blood using the Wizard ${ }^{\circledR}$ Genomic DNA Purification Kit (Promega, Madison, WI, USA) according to the manufacturer's instructions. Briefly, white blood cells were lysed, thereafter cellular proteins were removed by salt precipitation, and high molecular weight genomic DNA left in solution was then concentrated and desalted by isopropanol precipitation. A total of 566 participants $(11.7 \%$ males) who consented to genetic analyses were genotyped for six single nucleotide polymorphisms (SNPs) that have been shown to confer susceptibility to T2DM in previous studies elsewhere: ENPP1-rs997509 and -rs1044498, FTO-9941349 and -rs3751812, TCF7L2-rs12255372 and -rs7903146. The SNPs were genotyped using their corresponding Taqman genotyping assays (Applied Biosystems, USA) on a BioRad Optica (Biorad, USA) and validated by sequencing, giving $100 \%$ genotyping concordance for all samples that were repeated.

\section{Statistical analysis}

General characteristics of the study group are sum- marized as count and percentage for dichotomous traits, mean and standard deviation (SD) or median and $25^{\text {th }}-75^{\text {th }}$ percentiles for quantitative traits. Traits were log-transformed to approximate normality, where necessary, prior to analysis. SNPs were tested for departure from Hardy-Weindberg Equilibrium (HWE) expectation via a chi square goodness of fit test. Linkage disequilibrium (LD) was estimated using the D' statistic. Logistic regression models were used for the analysis of dichotomous traits, assuming both recessive and log-additive models for the SNPs. We investigated the association of each SNP with each trait, overall and tested for heterogeneity by major subgroups by adding the interaction term of major grouping variables and each SNP to a model that contained the main effects of grouping variable and the relevant SNP. Results corresponding to p-values below $5 \%$ are described as significant. Adjustment for multiple testing was conducted via Bonferroni methods. All analyses used the statistical software R (version 3.0.0 [2013-04-03], The R Foundation for statistical computing, Vienna, Austria). SNPs analyses used the packages 'genetic', 'gap', 'SNPassoc' and 'hapassoc'.

\section{Results \\ Baseline characteristics}

Of 566 participants that consented for genetic studies, 480 had complete data for analysis and their clinical characteristics are summarized in Table 1 . One hundred and fifty eight $(32 \%)$ individuals had T2DM. As expected, T2DM-related traits differed significantly according to diabetes status. For example, significant differences in the distribution of insulin resistance/sensitivity indicators were observed between the two groups (all $\mathrm{p}$ $<0.0001$, except for glucose/insulin ratio $(\mathrm{p}=0.009)$. Participants with diabetes compared to non-diabetics, were older ( $p<0.0001)$, had higher body mass index $(p=0.023)$, higher waist circumference and waist-to-hip ratio (both $\mathrm{p}<0.0001$ ), higher systolic blood pressure (130 vs. $121 \mathrm{mmHg}, \mathrm{p}<0.0001)$, and consisted of a higher proportion of individuals with hypertension $(77.6 \%$ vs. $67.1 \%, p=0.018)$. Furthermore, diabetic individuals had higher triglycerides $(\mathrm{p}<0.0001)$. 
Table 1 - Baseline characteristics overall and by body mass index and diabetes status

\begin{tabular}{|c|c|c|c|c|c|c|c|c|}
\hline Characteristics & $\begin{array}{l}\text { No } \\
\text { diabetes }\end{array}$ & Diabetes & $\begin{array}{l}\text { p- } \\
\text { value }\end{array}$ & $\begin{array}{l}\text { Normal } \\
\text { weight }\end{array}$ & $\begin{array}{l}\text { overweig } \\
\text { ht }\end{array}$ & obese & $\begin{array}{l}\text { p- } \\
\text { value }\end{array}$ & Overall \\
\hline $\mathrm{N}$ & 328 & 152 & & 118 & 129 & 233 & & 480 \\
\hline Gender, male $\mathrm{n}(\%)$ & $18(5.5)$ & $34(22.4)$ & $\begin{array}{l}<0.000 \\
1\end{array}$ & $16(13.6)$ & $17(13.2)$ & $19(8.1)$ & 0.185 & $52(10.8)$ \\
\hline Mean Age, year (SD) & $\begin{array}{l}53.2 \\
(13.5)\end{array}$ & $60.1(11.8)$ & $\begin{array}{l}<0.000 \\
1\end{array}$ & $\begin{array}{l}54.7 \\
(15.0)\end{array}$ & $\begin{array}{l}55.9 \\
(12.8)\end{array}$ & $\begin{array}{l}55.5 \\
(12.9)\end{array}$ & 0.793 & $\begin{array}{l}55.4 \\
(13.4)\end{array}$ \\
\hline $\begin{array}{l}\text { Mean Systolic blood pressure, } \\
\mathrm{mmHg}(\mathrm{SD})\end{array}$ & $121(18)$ & $130(22)$ & $\begin{array}{l}<0.000 \\
1\end{array}$ & $119(22)$ & $122(17)$ & $127(20)$ & 0.0007 & $124(20)$ \\
\hline $\begin{array}{l}\text { Mean Diastolic blood pressure, } \\
\mathrm{mmHg}(\mathrm{SD})\end{array}$ & $74(12)$ & $77(16)$ & 0.076 & $73(18)$ & $74(11)$ & $77(12)$ & 0.016 & $75(14)$ \\
\hline Hypertension, n (\%) & $\begin{array}{l}220 \\
(67.1)\end{array}$ & $118(77.6)$ & 0.018 & $64(54.2)$ & $93(72.1)$ & $181(77.7)$ & $\begin{array}{l}<0.000 \\
1\end{array}$ & $338(70.4)$ \\
\hline $\begin{array}{l}\text { Mean Body mass index, } \mathrm{kg} / \mathrm{m}^{2} \\
\text { (SD) }\end{array}$ & $30.1(7.2)$ & $31.9(7.2)$ & 0.012 & $22.0(2.3)$ & $27.7(1.4)$ & $36.5(5.8)$ & $\begin{array}{l}<0.000 \\
1\end{array}$ & $30.6(7.4)$ \\
\hline $\begin{array}{l}\text { Mean Waist circumference, } \mathrm{cm} \\
\text { (SD) }\end{array}$ & $96(15)$ & $103(14)$ & $\begin{array}{l}<0.000 \\
1\end{array}$ & $81(10)$ & $93(6)$ & $109(11)$ & $\begin{array}{l}<0.000 \\
1\end{array}$ & $98(15)$ \\
\hline $\begin{array}{l}\text { Mean Hip circumference, } \mathrm{cm} \\
\text { (SD) }\end{array}$ & $111(15)$ & $112(16)$ & 0.558 & $96(9)$ & $106(6)$ & $122(13)$ & $\begin{array}{l}<0.000 \\
1\end{array}$ & $111(15)$ \\
\hline Mean Waist/hip ratio, (SD) & $\begin{array}{l}0.86 \\
(0.08)\end{array}$ & $0.92(0.08)$ & $\begin{array}{l}<0.000 \\
1\end{array}$ & $\begin{array}{l}0.84 \\
(0.07)\end{array}$ & $\begin{array}{l}0.89 \\
(0.07)\end{array}$ & $0.89(0.08)$ & $\begin{array}{l}<0.000 \\
1\end{array}$ & $\begin{array}{l}0.88 \\
(0.08)\end{array}$ \\
\hline Mean HbAlc, \% (SD) & $5.7(0.4)$ & $7.8(1.9)$ & $\begin{array}{l}<0.000 \\
1\end{array}$ & $5.8(0.9)$ & $6.6(1.8)$ & $6.4(1.3)$ & $\begin{array}{l}<0.000 \\
1\end{array}$ & $6.3(1.4)$ \\
\hline $\begin{array}{l}\text { Mean Fasting blood glucose, } \\
\mathrm{mmol} / \mathrm{l} \text { (SD) }\end{array}$ & $5.3(0.7)$ & $9.5(3.8)$ & $\begin{array}{l}<0.000 \\
1\end{array}$ & $5.9(2.1)$ & $7.0(3.8)$ & $6.8(2.8)$ & 0.007 & $6.6(3.0)$ \\
\hline Mean $2 \mathrm{~h}$ glucose, $\mathrm{mmol} / \mathrm{l}(\mathrm{SD})$ & $6.8(1.7)$ & $13.4(4.7)$ & $\begin{array}{l}<0.000 \\
1\end{array}$ & $7.2(2.5)$ & $7.7(3.6)$ & $8.7(3.9)$ & 0.0008 & $8.1(3.5)$ \\
\hline $\begin{array}{l}\text { Mean Triglycerides, mmol/l } \\
\text { (SD) }\end{array}$ & $1.4(1.0)$ & $1.8(0.9)$ & $\begin{array}{l}<0.000 \\
1\end{array}$ & $1.2(1.0)$ & $1.6(1.1)$ & $1.6(0.8)$ & & \\
\hline $\begin{array}{l}\text { Mean HDL cholesterol, mmol/1 } \\
\text { (SD) }\end{array}$ & $1.3(0.3)$ & $1.2(0.3)$ & 0.0001 & $1.4(0.4)$ & $1.3(0.3)$ & $1.2(0.3)$ & $\begin{array}{l}<0.000 \\
1\end{array}$ & $1.3(0.3)$ \\
\hline $\begin{array}{l}\text { Mean LDL cholesterol, mmol/1 } \\
\text { (SD) }\end{array}$ & $3.8(1.0)$ & $3.8(1.1)$ & 0.984 & $3.6(0.9)$ & $3.8(1.0)$ & $3.8(1.0)$ & 0.075 & $3.8(1.0)$ \\
\hline $\begin{array}{l}\text { Mean Total cholesterol, mmol/1 } \\
\text { (SD) }\end{array}$ & $5.7(1.2)$ & $5.8(1.2)$ & 0.582 & $5.5(1.1)$ & $5.8(1.2)$ & $5.8(1.2)$ & 0.084 & $5.7(1.2)$ \\
\hline $\begin{array}{l}\text { Median Insulin mmol/1(25th- } \\
\text { 75th percentiles) }\end{array}$ & $\begin{array}{l}6.9[2.9- \\
12.2]\end{array}$ & $8.7[2.8-14.8]$ & 0.043 & $\begin{array}{l}3.7[1.5- \\
8.0]\end{array}$ & $\begin{array}{l}7.0[3.2- \\
11.1]\end{array}$ & $\begin{array}{l}9.1[4.9- \\
15.0]\end{array}$ & $\begin{array}{l}<0.000 \\
1\end{array}$ & $\begin{array}{l}7.4[2.9- \\
13.2]\end{array}$ \\
\hline $\begin{array}{l}\text { Median } 2 \mathrm{~h} \text { insulin } \mathrm{mmol} / \mathrm{l}(25 \text { th- } \\
75 \text { th percentiles })\end{array}$ & $\begin{array}{l}39.5 \\
{[21.7-} \\
67.4]\end{array}$ & $\begin{array}{l}54.9[21.8- \\
116.1]\end{array}$ & 0.026 & $\begin{array}{l}28.8 \\
{[17.1-} \\
45.2]\end{array}$ & $\begin{array}{l}36.7 \\
{[22.7-} \\
61.0]\end{array}$ & $\begin{array}{l}56.5 \\
{[31.0-} \\
106.4]\end{array}$ & $\begin{array}{l}<0.000 \\
1\end{array}$ & \\
\hline $\begin{array}{l}\text { Median Glucose/insulin ( } 25 \text { th- } \\
\text { 75th percentiles) }\end{array}$ & $\begin{array}{l}0.75 \\
{[0.44-} \\
1.74]\end{array}$ & $\begin{array}{l}1.03[0.53- \\
2.71]\end{array}$ & 0.025 & $\begin{array}{l}1.37 \\
{[0.66-} \\
3.63]\end{array}$ & $\begin{array}{l}0.81 \\
{[0.53-} \\
1.95]\end{array}$ & $\begin{array}{l}0.67 \\
{[0.40-} \\
1.49]\end{array}$ & $\begin{array}{l}<0.000 \\
1\end{array}$ & $\begin{array}{l}0.78[0.48- \\
1.95]\end{array}$ \\
\hline $\begin{array}{l}\text { Median HOMA-IR (25th-75th } \\
\text { percentiles) }\end{array}$ & $\begin{array}{l}1.56 \\
{[0.60-} \\
2.91]\end{array}$ & $\begin{array}{l}3.11[1.21- \\
5.89]\end{array}$ & $\begin{array}{l}<0.000 \\
1\end{array}$ & $\begin{array}{l}0.95 \\
{[0.35-} \\
2.11]\end{array}$ & $\begin{array}{l}1.90 \\
{[0.80-} \\
3.35]\end{array}$ & $\begin{array}{l}2.62 \\
{[1.20-} \\
4.69]\end{array}$ & $\begin{array}{l}<0.000 \\
1\end{array}$ & $\begin{array}{l}1.85 \\
{[0.76-} \\
3.71]\end{array}$ \\
\hline $\begin{array}{l}\text { Median HOMA-B } \% \text { (25th-75th } \\
\text { percentiles) }\end{array}$ & $\begin{array}{l}75.4 \\
{[35.6-} \\
149.0]\end{array}$ & $\begin{array}{l}34.8[9.4- \\
71.9]\end{array}$ & $\begin{array}{l}<0.000 \\
1\end{array}$ & $\begin{array}{l}43.0 \\
{[15.2-} \\
97.1]\end{array}$ & $\begin{array}{l}56.2 \\
{[15.1-} \\
96.8]\end{array}$ & $\begin{array}{l}75.4 \\
{[31.2-} \\
142.9]\end{array}$ & 0.001 & $\begin{array}{l}60.0 \\
{[20.0-} \\
117.5]\end{array}$ \\
\hline $\begin{array}{l}\text { Median QUICKI (25th-75th } \\
\text { percentiles) }\end{array}$ & $\begin{array}{l}0.15 \\
{[0.14-} \\
0.18]\end{array}$ & $\begin{array}{l}0.14[0.13- \\
0.16]\end{array}$ & $\begin{array}{l}<0.000 \\
1\end{array}$ & $\begin{array}{l}0.17 \\
{[0.15-} \\
0.20]\end{array}$ & $\begin{array}{l}0.15 \\
{[0.14-} \\
0.17]\end{array}$ & $\begin{array}{l}0.14 \\
{[0.13-} \\
0.16]\end{array}$ & $\begin{array}{l}<0.000 \\
1\end{array}$ & $\begin{array}{l}0.15 \\
{[0.14-} \\
0.17]\end{array}$ \\
\hline $\begin{array}{l}\text { Median FIRI (25th-75th } \\
\text { percentiles) }\end{array}$ & $\begin{array}{l}1.40 \\
{[0.54-} \\
2.62]\end{array}$ & $\begin{array}{l}2.80[1.09- \\
5.30]\end{array}$ & $\begin{array}{l}<0.000 \\
1\end{array}$ & $\begin{array}{l}0.85 \\
{[0.31-} \\
1.90]\end{array}$ & $\begin{array}{l}1.71 \\
{[0.72-} \\
3.01]\end{array}$ & $\begin{array}{l}2.36 \\
{[1.08-} \\
4.22]\end{array}$ & $\begin{array}{l}<0.000 \\
1\end{array}$ & $\begin{array}{l}1.66 \\
{[0.68-} \\
3.34]\end{array}$ \\
\hline
\end{tabular}

Abbreviations: CRP, C-reactive protein; eGFR, estimated glomerularfiltration rate; FIRI, fasting insulin resistance index; HbA1C, glycated haemoglobin; HDL ,High Density Lipoproteins; HOMA-B\%, functional $\beta$-cells; GGT, $\gamma$-glutamyltransferase; LDL, Low Density Lipoproteins; HOMA-IR, homeostatic model assessment of insulin resistance; QUICKI, the quantitative insulin-sensitivity check index; SD, standard deviation.

Allele distribution and association test

Table 2 shows the allele and genotype distribution of SNPs investigated in the present study. None of the
SNPs investigated deviated from Hardy Weinberg Equilibrium (HWE) in the overall sample ( $p \geq 0.103)$. 
Table 2- Genotype distributions, minor allele frequencies, and unadjusted p-values for comparing genotype distributions according to diabetes and BMI status

\begin{tabular}{|c|c|c|c|c|c|c|c|c|}
\hline & No diabetes & Diabetes & p-value & $\begin{array}{l}\text { Normal } \\
\text { weight }\end{array}$ & overweight & Obese & $\begin{array}{l}\text { p- } \\
\text { value }\end{array}$ & Overall \\
\hline $\mathrm{N}$ & 328 & 152 & & 118 & 129 & 233 & & 532 \\
\hline \multicolumn{9}{|l|}{ ENPP1-rs997509 } \\
\hline $\mathrm{C} / \mathrm{C}, \mathrm{n}(\%)$ & $254(77.4)$ & $121(79.6)$ & 0.186 & $\begin{array}{l}97 \\
(82.2)\end{array}$ & $94(72.9)$ & $\begin{array}{l}184 \\
(79.0)\end{array}$ & 0.342 & $\begin{array}{l}375 \\
(78.1)\end{array}$ \\
\hline $\mathrm{C} / \mathrm{T}, \mathrm{n}(\%)$ & $70(21.3)$ & $26(17.1)$ & & $\begin{array}{l}28 \\
(15.2)\end{array}$ & $33(25.6)$ & $\begin{array}{l}45 \\
(19.3)\end{array}$ & & $\begin{array}{l}96 \\
(20.0)\end{array}$ \\
\hline $\mathrm{T} / \mathrm{T}, \mathrm{n}(\%)$ & $4(1.2)$ & $5(3.3)$ & & $3(2.5)$ & $2(1.5)$ & $4(1.7)$ & & $9(1.9)$ \\
\hline $\mathrm{T}, \mathrm{n}(\%)$ & 78 (11.9) & $36(11.8)$ & 0.920 & $\begin{array}{l}34 \\
(14.4)\end{array}$ & $37(14.3)$ & $\begin{array}{l}53 \\
(11.4)\end{array}$ & 0.383 & $\begin{array}{l}114 \\
(11.9)\end{array}$ \\
\hline HWE (p-value) & $>0.999$ & 0.038 & & 0.095 & $>0.999$ & 0.511 & & 0.379 \\
\hline \multicolumn{9}{|c|}{ ENPP1-rs1044498 } \\
\hline $\mathrm{C} / \mathrm{C}, \mathrm{n}(\%)$ & $81(24.7)$ & $41(27.0)$ & 0.548 & $\begin{array}{l}33 \\
(28.0)\end{array}$ & $35(25.6)$ & $\begin{array}{l}56 \\
(24.0)\end{array}$ & 0.852 & $\begin{array}{l}122 \\
(25.0)\end{array}$ \\
\hline $\mathrm{C} / \mathrm{A}, \mathrm{n}(\%)$ & $175(53.4)$ & $73(48.0)$ & & $\begin{array}{l}62 \\
(52.5)\end{array}$ & $66(51.2)$ & $\begin{array}{l}120 \\
(51.5)\end{array}$ & & $\begin{array}{l}248 \\
(51.7)\end{array}$ \\
\hline $\mathrm{A} / \mathrm{A}, \mathrm{n}(\%)$ & $72(21.9)$ & $38(25.0)$ & & $\begin{array}{l}23 \\
(19.5)\end{array}$ & $30(23.3)$ & $\begin{array}{l}57 \\
(24.5)\end{array}$ & & $\begin{array}{l}110 \\
(22.9)\end{array}$ \\
\hline $\mathrm{A}, \mathrm{n}(\%)$ & $319(48.6)$ & $149(49.0)$ & $>0.999$ & $\begin{array}{l}108 \\
(45.8)\end{array}$ & $126(48.8)$ & $\begin{array}{l}234 \\
(50.2)\end{array}$ & 0.538 & $\begin{array}{l}468 \\
(48.8)\end{array}$ \\
\hline HWE (p-value) & 0.269 & 0.629 & & 0.582 & 0.861 & 0.695 & & 0.522 \\
\hline \multicolumn{9}{|l|}{ FTO-9941349 } \\
\hline $\mathrm{C} / \mathrm{C}, \mathrm{n}(\%)$ & $187(57.0)$ & $73(48.0)$ & 0.185 & $\begin{array}{l}63 \\
(53.4)\end{array}$ & $64(49.6)$ & $\begin{array}{l}133 \\
(57.1)\end{array}$ & 0.595 & $\begin{array}{l}260 \\
(54.2)\end{array}$ \\
\hline $\mathrm{C} / \mathrm{T}, \mathrm{n}(\%)$ & $118(36.0)$ & $66(43.4)$ & & $\begin{array}{l}44 \\
(37.3)\end{array}$ & $56(43.4)$ & $\begin{array}{l}84 \\
(36.0)\end{array}$ & & $\begin{array}{l}184 \\
(38.3)\end{array}$ \\
\hline $\mathrm{T} / \mathrm{T}, \mathrm{n}(\%)$ & $23(7.0)$ & $13(8.5)$ & & $11(9.3)$ & $9(7.0)$ & $\begin{array}{l}16 \\
(6.9)\end{array}$ & & $36(7.5)$ \\
\hline $\mathrm{T}, \mathrm{n}(\%)$ & $164(25.0)$ & $92(30.3)$ & 0.102 & $\begin{array}{l}66 \\
(28.0)\end{array}$ & $74(28.7)$ & $\begin{array}{l}116 \\
(24.9)\end{array}$ & 0.475 & $\begin{array}{l}256 \\
(26.7)\end{array}$ \\
\hline HWE (p-value) & 0.463 & 0.848 & & 0.492 & 0.666 & 0.599 & & 0.642 \\
\hline \multicolumn{9}{|l|}{ FTO-rs3751812 } \\
\hline $\mathrm{G} / \mathrm{G}, \mathrm{n}(\%)$ & $218(66.5)$ & $93(61.2)$ & 0.516 & $\begin{array}{l}75 \\
(63.6)\end{array}$ & $79(61.2)$ & $\begin{array}{l}157 \\
(67.4)\end{array}$ & 0.761 & $\begin{array}{l}311 \\
(64.8)\end{array}$ \\
\hline $\mathrm{G} / \mathrm{T}, \mathrm{n}(\%)$ & $100(30.5)$ & $53(34.9)$ & & $\begin{array}{l}38 \\
(32.2)\end{array}$ & $46(35.7)$ & $\begin{array}{l}69 \\
(29.6)\end{array}$ & & $\begin{array}{l}153 \\
(31.9)\end{array}$ \\
\hline $\mathrm{T} / \mathrm{T}, \mathrm{n}(\%)$ & $10(3.0)$ & $6(3.9)$ & & $5(4.2)$ & $4(3.1)$ & $7(3.0)$ & & $16(3.3)$ \\
\hline $\mathrm{T}, \mathrm{n}(\%)$ & $120(18.3)$ & $65(21.4)$ & 0.299 & $\begin{array}{l}48 \\
(20.3)\end{array}$ & $54(20.9)$ & $\begin{array}{l}83 \\
(17.8)\end{array}$ & 0.530 & $\begin{array}{l}185 \\
(19.3)\end{array}$ \\
\hline HWE (p-value) & 0.854 & 0.810 & & $>0.999$ & 0.593 & $>0.999$ & & 0.662 \\
\hline \multicolumn{9}{|c|}{$T C F 7 L 2-r s 12255372$} \\
\hline $\mathrm{G} / \mathrm{G}, \mathrm{n}(\%)$ & $221(67.4)$ & $92(60.5)$ & 0.305 & $\begin{array}{l}72 \\
(61.0)\end{array}$ & $88(68.2)$ & $\begin{array}{l}153 \\
(65.7)\end{array}$ & 0.623 & $\begin{array}{l}313 \\
(65.2)\end{array}$ \\
\hline $\mathrm{G} / \mathrm{T}, \mathrm{n}(\%)$ & $92(28.0)$ & $50(32.9)$ & & $\begin{array}{l}37 \\
(31.6)\end{array}$ & $36(27.9)$ & $\begin{array}{l}69 \\
(29.6)\end{array}$ & & $\begin{array}{l}142 \\
(29.6)\end{array}$ \\
\hline $\mathrm{T} / \mathrm{T}, \mathrm{n}(\%)$ & $15(4.6)$ & $10(6.6)$ & & $9(7.6)$ & $5(3.9)$ & $\begin{array}{l}11 \\
(4.7)\end{array}$ & & $25(5.2)$ \\
\hline $\mathrm{T}, \mathrm{n}(\%)$ & $122(18.6)$ & $70(23.0)$ & 0.131 & $\begin{array}{l}55 \\
(23.3)\end{array}$ & $46(17.8)$ & $\begin{array}{l}91 \\
(19.5)\end{array}$ & 0.295 & $\begin{array}{l}192 \\
(20.0)\end{array}$ \\
\hline HWE (p-value) & 0.200 & 0.364 & & 0.197 & 0.552 & 0.403 & & 0.115 \\
\hline \multicolumn{9}{|c|}{ TCF7L2-rs7903146 } \\
\hline $\mathrm{C} / \mathrm{C}, \mathrm{n}(\%)$ & $184(56.1)$ & $66(43.4)$ & 0.025 & $\begin{array}{l}57 \\
(48.3)\end{array}$ & $66(51.2)$ & $\begin{array}{l}127 \\
(54.5)\end{array}$ & 0.330 & $\begin{array}{l}250 \\
(52.1)\end{array}$ \\
\hline $\mathrm{C} / \mathrm{T}, \mathrm{n}(\%)$ & $129(39.3)$ & $74(48.7)$ & & $\begin{array}{l}52 \\
(44.0)\end{array}$ & $53(41.1)$ & $\begin{array}{l}98 \\
(42.1)\end{array}$ & & $\begin{array}{l}203 \\
(42.3)\end{array}$ \\
\hline $\mathrm{T} / \mathrm{T}, \mathrm{n}(\%)$ & $15(4.6)$ & $12(7.9)$ & & $9(7.6)$ & $10(7.7)$ & $8(3.4)$ & & $27(5.6)$ \\
\hline $\mathrm{T}, \mathrm{n}(\%)$ & $159(24.2)$ & $98(32.2)$ & 0.011 & $\begin{array}{l}70 \\
(29.7)\end{array}$ & $73(28.3)$ & $\begin{array}{l}114 \\
(24.5)\end{array}$ & 0.275 & $\begin{array}{l}257 \\
(26.8)\end{array}$ \\
\hline HWE (p-value) & 0.231 & 0.196 & & 0.661 & $>0.999$ & 0.050 & & 0.103 \\
\hline
\end{tabular}

Within each gene, the SNPs were in linkage disequilibrium (LD) in all sub-groups of the study population (D' $\geq 0.614$ ) (online Figure 1). The SNPs investigated showed no differences in the distribution of alleles and genotypes between the study population sub-groups according to T2DM and BMI status, except rs7903146. The TCF7L2-rs7903146 minor allele was prevalent in diabetic compared to non-diabetic individuals $(32.2 \%$ vs. $24.2 \%, \mathrm{p}=0.011)$. 


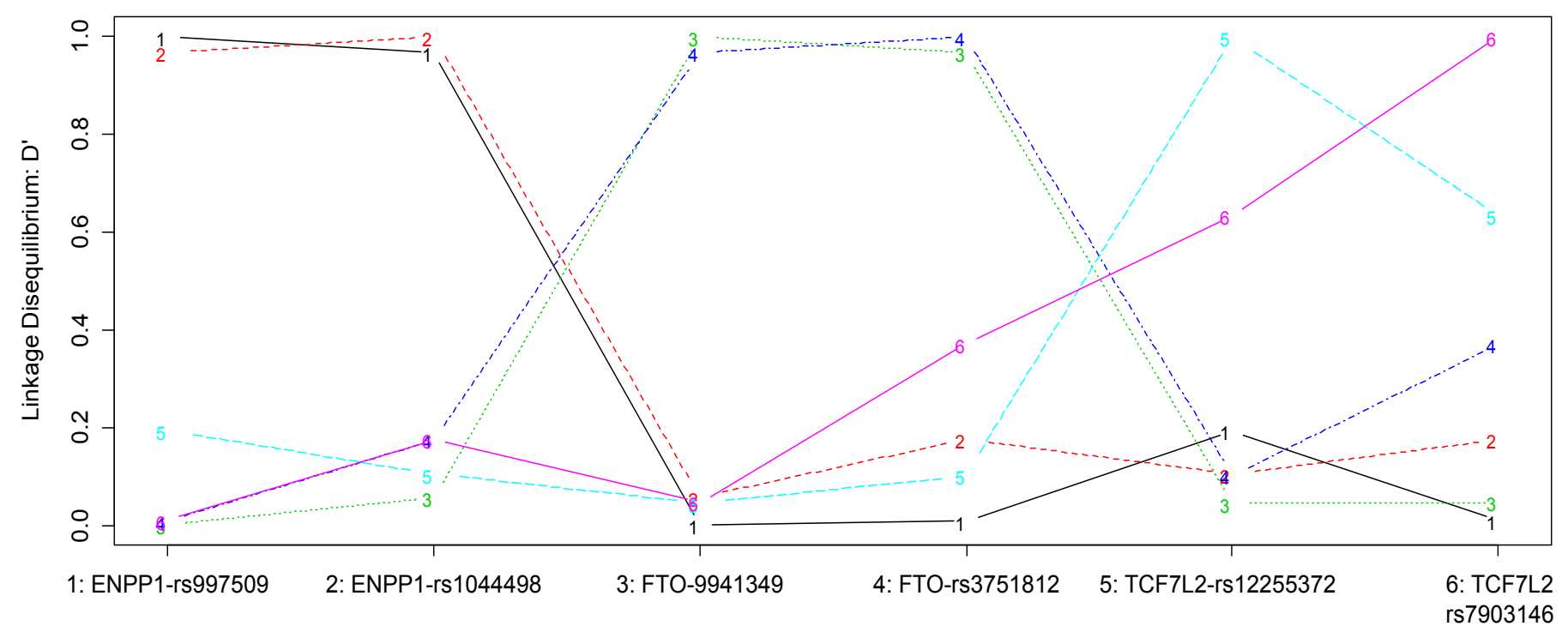

Linkage Disequilibrium Table

\begin{tabular}{|c|c|c|c|c|c|}
\hline & ENPP1-rs1044498 & FTO-9941349 & FTO-rs3751812 & TCF7L2-rs12255372 & TCF7L2-rs7903146 \\
\hline ENPP1-rs997509 & $\begin{array}{l}0.050042 \\
0.96806 \\
-0.34659 \\
1.156+02 \\
<2-16 \\
480 \\
480\end{array}$ & $\begin{array}{c}0.000194 \\
0.00222 \\
0.00135 \\
1.766-03 \\
0.966538 \\
480 \\
\end{array}$ & $\begin{array}{c}.001005 \\
0.01049 \\
0.00788 \\
5.96 \mathrm{e}-02 \\
0.807149 \\
480\end{array}$ & $\begin{array}{c}-0.004604 \\
0.19384 \\
-0.03558 \\
1.22 e+00 \\
0.270310 \\
480 \\
\end{array}$ & $\begin{array}{c}0.001069 \\
0.01229 \\
0.00746 \\
5.35 \mathrm{e}-02 \\
0.817129 \\
480\end{array}$ \\
\hline ENPP1-rs1044498 & & $\begin{array}{l}0.007978 \\
0.05388 \\
0.03609 \\
1.250+00 \\
0.263432 \\
480\end{array}$ & $\begin{array}{l}0.017448 \\
0.17666 \\
0.08850 \\
7.52+000 \\
0.006105 \\
480\end{array}$ & $\begin{array}{l}-0.010362 \\
0.10627 \\
-0.05182 \\
2.58+000 \\
0.108335 \\
480\end{array}$ & $\begin{array}{l}-0.022990 \\
0.17616 \\
-0.10388 \\
1.04+0101 \\
0.001288 \\
480\end{array}$ \\
\hline FTO-9941349 & & & $\begin{array}{c}0.136806 \\
0.96806 \\
0.78434 \\
5.91 \mathrm{e}+02 \\
<2 \mathrm{e}-16 \\
480\end{array}$ & $\begin{array}{c}0.006829 \\
0.04656 \\
0.03860 \\
1.43 e+00 \\
0.231658 \\
480\end{array}$ & $\begin{array}{c}-0.003437 \\
0.04814 \\
-0.01755 \\
2.96 \mathrm{e}-01 \\
0.586555 \\
480\end{array}$ \\
\hline FTO-rs3751812 & & & & $\begin{array}{c}-0.003950 \\
0.10249 \\
-0.02504 \\
6.02 \mathrm{e}-01 \\
0.437900 \\
480 \\
\end{array}$ & $\begin{array}{l}-0.017258 \\
-0.132927 \\
-1.170+01 \\
0.0006034 \\
080 \\
\end{array}$ \\
\hline TCF7L2-rs12255372 & $\begin{array}{c}D \\
D^{\prime} \\
r \\
x^{2} \\
P-x^{2} \text { alue } \\
n\end{array}$ & & & & $\begin{array}{l}0.093021 \\
0.635544 \\
.552523 \\
2.65+02+02 \\
<2+16 \\
480\end{array}$ \\
\hline
\end{tabular}

Online Figure 1 - linkage disequilibrium plot (upper panel) and linkage disequilibrium table (lower panel) for the six SNP in 480 participants. ENPP1-rs997509 is in linkage disequilibrium (LD) with ENPP1-rs1044498 overall ( $\left(\mathrm{D}^{\prime}=0.968\right)$, in participants without diabetes $\left(\mathrm{D}^{\prime}=0.950\right)$, in those with diabetes $\left(\mathrm{D}^{\prime}=0.999\right)$, in normal weight $\left(\mathrm{D}^{\prime}=0.998\right)$, overweight $\left(\mathrm{D}^{\prime}=0.999\right)$ and obese participants $\left(\mathrm{D}^{\prime}=0.939\right) . F T O-9941349$ is in linkage disequilibrium (LD) with $F T O-\mathbf{r s 3 7 5 1 8 1 2}$ overall $\left(D^{\prime}=0.968\right)$, in participants without diabetes $\left(\mathrm{D}^{\prime}=0.952\right)$ and in those with diabetes $\left(\mathrm{D}^{\prime}>0.999\right)$, in normal weight $\left(\mathrm{D}^{\prime}=0.937\right)$, overweight $\left(\mathrm{D}^{\prime}=0.943\right)$ and obese participants $\left(\mathrm{D}^{\prime}=0.999\right)$. TCF7L2rs12255372 is in linkage disequilibrium (LD) with $T C F 7 L 2-$ rs7903146 overall $\left(\mathrm{D}^{\prime}=0.635\right)$, in participants without diabetes $\left(D^{\prime}=0.648\right)$ and in those with diabetes $\left(D^{\prime}=0.602\right)$, in normal weight $\left(D^{\prime}=0.614\right)$, overweight $\left(D^{\prime}=0.648\right)$ and obese participants $\left(D^{\prime}=0.641\right)$. 
In linear analyses adjusted for age, sex, BMI and HOMA IR (Table 3), minor allele (T) of ENPP1-rs997509 was associated with a higher risk of prevalent type 2 diabetes under a recessive model [odds ratio (95\% confidence interval), 4.60 (1.07 to 19.86); $\mathrm{p}=0.040$ ], but with no evidence of significant statistical interaction with BMI categories. The TCF7L2-rs7903146 minor allele showed a borderline significant association with a high risk of T2DM [1.43 (1.00 to 2.04); $\mathrm{p}=0.053]$ in log-additive models (Table 3 ).

Table 3 -logistic regression models showing the effects of genes on prevalent diabetes risk.

\begin{tabular}{|c|c|c|c|c|}
\hline \multirow[b]{2}{*}{ SNP } & \multirow[b]{2}{*}{ Allele } & \multicolumn{2}{|l|}{ Overall } & \multirow{2}{*}{ BMI categories*SNP interaction } \\
\hline & & Effects size $(95 \% \mathrm{CI})$ & $\mathbf{p}$ & \\
\hline \multicolumn{5}{|l|}{ Recessive model } \\
\hline ENPP1-rs997509 & $\mathrm{T} / \mathrm{T}$ & $4.60(1.07$ to 19.86$)$ & 0.040 & 0.173 \\
\hline ENPP1-rs1044498 & $\mathrm{A} / \mathrm{A}$ & 1.48 (0.89 to 2.47$)$ & 0.134 & 0.059 \\
\hline FTO-9941349 & $\mathrm{T} / \mathrm{T}$ & $1.10(0.49$ to 2.43$)$ & 0.822 & 0.155 \\
\hline FTO-rs3751812 & $\mathrm{T} / \mathrm{T}$ & $0.85(0.26$ to 2.81$)$ & 0.937 & 0.374 \\
\hline TCF7L2-rs 12255372 & $\mathrm{~T} / \mathrm{T}$ & $1.56(0.61$ to 4.00$)$ & 0.361 & 0.160 \\
\hline$T C F 7 L 2-r s 7903146$ & $\mathrm{~T} / \mathrm{T}$ & $1.57(0.64$ to 3.86$)$ & 0.329 & 0.972 \\
\hline \multicolumn{5}{|l|}{ Log additive model } \\
\hline ENPP1-rs997509 & $\mathrm{T}$ & $0.98(0.62$ to 1.54$)$ & 0.915 & \\
\hline ENPP1-rs1044498 & A & $0.99(0.72$ to 1.36$)$ & 0.954 & \\
\hline FTO-9941349 & $\mathrm{T}$ & $1.40(1.00$ to 1.96$)$ & 0.052 & \\
\hline FTO-rs3751812 & $\mathrm{T}$ & $1.22(0.83$ to 1.80$)$ & 0.307 & \\
\hline TCF7L2-rs 12255372 & $\mathrm{~T}$ & $1.28(0.89$ to 1.83$)$ & 0.191 & \\
\hline TCF7L2-rs7903146 & $\mathrm{T}$ & $1.43(1.00$ to 2.04$)$ & 0.053 & \\
\hline
\end{tabular}

Models are adjusted for age, sex, BMI and HOMA IR. Effect estimates are odd ratio and 95\% confidence intervals. Abbreviation: ENPP1, ectonucleotide pyrophosphatase/phosphodiesterase gene; $\boldsymbol{F T O}$, fat mass and obesity-associated gene; $\boldsymbol{T C F} \mathbf{L} \mathbf{2}$, transcription factor 7-like 2 gene.

Although the distribution of alleles and genotypes of the FTO-rs9941349 were not significant between the subgroups (Table 2), the minor allele was marginally significantly associated with a high risk of T2DM [1.40 (1.00 to 1.96); $\mathrm{p}=0.052$ ] in log-additive models (Table
3). The inferred haplotype association analysis showed a negative effect of the rs997509-rs1044498 (TA) and rs9941349- rs3751812 (CT) on the risk of T2DM, however, the haplotypes occurred at very low frequencies (Table 4). 


\begin{tabular}{|c|c|c|c|c|c|}
\hline \multirow[b]{2}{*}{ SNP 1} & \multirow[b]{2}{*}{ SNP 2} & \multirow[b]{2}{*}{$\begin{array}{l}\text { Haplotyp } \\
\text { e }\end{array}$} & \multirow[b]{2}{*}{$\begin{array}{l}\text { Estimate } \\
\text { d } \\
\text { frequency } \\
(\%)\end{array}$} & \multicolumn{2}{|l|}{ Overall } \\
\hline & & & & Effects size $(95 \% \mathrm{CI})$ & $\mathbf{p}$ \\
\hline \multirow[t]{5}{*}{ ENPP1-rs997509 } & ENPP1-rs1044498 & $\mathrm{CA}$ & 48.4 & 1 (reference) & \\
\hline & & $\mathrm{CC}$ & 39.6 & $0.98(0.70$ to 1.38$)$ & 0.904 \\
\hline & & $\mathrm{TA}$ & 0.2 & $\begin{array}{l}7.213^{(-10)}\left[7.213^{(-10)} \text { to } 7.213^{(-}\right. \\
\left.{ }^{(-)}\right]\end{array}$ & $\begin{array}{l}<0.000 \\
1\end{array}$ \\
\hline & & $\mathrm{TC}$ & 11.8 & $1.01(0.62$ to 1.65$)$ & 0.969 \\
\hline & Global effects & & & & 0.753 \\
\hline \multirow[t]{4}{*}{ FTO-rs9941349 } & FTO-rs3751812 & CG & 73.1 & 1 (reference) & \\
\hline & & $\mathrm{CT}$ & 0.5 & $4.407^{(-9)}\left[4.407^{(-9)}\right.$ to $\left.4.407^{(-9)}\right]$ & $\begin{array}{l}<0.000 \\
1\end{array}$ \\
\hline & & $\mathrm{TG}$ & 7.6 & $1.52(0.88$ to 2.64$)$ & 0.135 \\
\hline & & $\mathrm{TT}$ & 18.8 & 1.34 (0.90 to 2.00$)$ & 0.150 \\
\hline \multirow[t]{4}{*}{$\begin{array}{l}\text { TCF7L2- } \\
\text { rs12255372 }\end{array}$} & $\begin{array}{l}\text { TCF7L2- } \\
\text { rs7903146 }\end{array}$ & GC & 67.8 & 1 (reference) & \\
\hline & & GT & 12.2 & $1.49(0.91$ to 2.45$)$ & 0.110 \\
\hline & & $\mathrm{TC}$ & 5.3 & $1.31(0.64$ to 2.73$)$ & 0.455 \\
\hline & & $\mathrm{TT}$ & 14.5 & $1.41(0.90$ to 2.20$)$ & 0.129 \\
\hline
\end{tabular}

Models are adjusted for age, sex, body mass index and HOMA-IR. Effect estimates are odd ratio and 95\% confidence intervals for qualitative traits. Abbreviation: ENPP1, ectonucleotide pyrophosphatase/phosphodiesterase

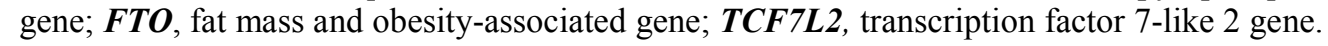

\section{Discussion}

In the present study we demonstrated that, in a recessive model, the minor allele of the ENPP1 rs997509 is associated with a high risk of T2DM. The presence of both ENPP1-rs997509 $\mathrm{T}$ alleles conferred a 7\% higher risk of prevalent T2DM. However, when analysed as a haplotype with rs1044498 (TA), the direction of association changed and this effect was abolished by the presence of the rs $1044498 \mathrm{C}$ allele. The haplotype association should be analysed with caution due to the low frequency of the TA haplotype $(0.2 \%)$. The rs 1044498 minor allele failed to show any association with T2DM risk, similar to studies conducted elsewhere in other ethnic population groups ${ }^{13,16}$. This is in contrast to findings of other studies conducted in Europeans and Chinese ${ }^{12,14}$ which demonstrated increased risk of T2DM among carriers of the 121QQ genotype.

The ENPP1-encoded protein inhibits insulin-signalling through interaction with and autophosphorylation of the insulin receptor beta-subunit, thus causing insulin resistance ${ }^{15}$. However, in the present study we did not find any significant association between ENPP1 SNPs and indicators of insulin resistance/sensitivity. Instead, in a previous study we demonstrated that the PPARGPro12 allele was associated with insulin resistance in this mixed ancestry cohort $^{21}$. Like ENPP1, PPARG plays a role in insulin sensitivity. Although the role of ENPP1 SNPs in insulin resistance was not evident in the present study, combined the findings of our two studies and based on the functions of the two proteins suggest that insulin resistance rather than a defect in insulin secretion is a primary defect that may lead to the development of T2DM in this population group. Further supporting this hypothesis is the marginal association between TCF7L2 and T2DM without any effect on the measure of beta-cell function (HOMA-B \%).

The two TCF7L2 polymorphisms have been reported to influence the development of T2DM across sever- 
al population groups worldwide ${ }^{30-32}$. While this is the case in other population groups, some studies reported a weak ${ }^{33,34}$ or no association ${ }^{35-38}$. Other studies highlighted the effect of the TCF7L2 polymorphisms on the progression of $\mathrm{T}_{2} \mathrm{DM}^{35,39}$, in some ethnic population groups these variants were associated with responses to medication ${ }^{40,41}$, and in others their effect on the disease was modulated by the type of $\operatorname{diet}^{42-44}$.Our results [1.43 (1.00 to 2.04), $\mathrm{p}=0.053$ ] are comparable to those reported for other African ethnic population groups ${ }^{17}$, ${ }^{18,20}$. It is possible that TCF7L2 polymorphisms have different effects in various ethnic population groups depending on the diet.

Insulin resistance is often related to obesity, and several studies have demonstrated that the association of ENPP1 and FTO polymorphisms with T2DM is mediated by obesity $8,9,45,46$. However, our study failed to show any association between ENPP1, FTO and obesity. The FTO-rs9941349 was marginally associated with an increased risk of T2DM independent of obesity in our study population. The finding that the rs9941349 polymorphism is marginally associated with the risk of T2DM raises the possibility that this SNP is in linkage disequilibrium with a causal locus that is yet to be identified in mixed ancestry population. In African-Americans, this polymorphism was positively associated with $\mathrm{BMI}^{47}$. It is possible that the different effects of the rs9941349 are attributable to different linkage disequilibrium blocks harbouring FTO polymorphisms in these two ethnic population groups. A comprehensive analysis of FTO polymorphisms in a large population of South African mixed-ancestry is warranted to clarify their effect on T2DM and obesity.

\section{Limitations}

Our study had limitations. We investigated for the first time the role of ENPP1, FTO and TCF7L2 polymorphisms in a South African mixed ancestry ethnic population with a unique genetic architecture. However, only two SNPs in each gene were studied, and therefore we cannot rule out the possible role of other variants within these genes. Due to financial constraints, ancestry informative markers were not used to account for population admixture, and there is a possibility that population admixture interfered with the association analysis. However, when tested none of the SNPs deviated from HWE. Association of ENPP1, FTO and TCF7L2 polymorphisms with high risk of T2DM may have also been confounded by lifestyle effects as reported elsewhere. For example, Moore and co-work$\mathrm{ers}^{48}$ showed that the significant association between ENPP1 and increased incidence of T2DM was mediated by lifestyle or metformin intervention. Ortega-Azorin and co-workers ${ }^{49}$ also found an interaction between FTO and mediterranean diet in determining T2DM, with carriers of the FTO-rs9939609 minor allele on a low Meddiet having a higher risk of prevalent T2DM than individuals homozygous for the major allele. In a European-American population group, Mattei and co-workers $^{50}$ observed a significant interaction between the TCF7L2-rs12255372-TT risk genotype and fat intake for changes in BMI, total fat mass, and trunk fat mass at 6 month of lifestyle intervention. The study suggested that this effect may help in inducing better glycemic control for such individuals predisposed to T2DM. It is therefore possible that the marginal association between FTO and TCF7L2 polymorphisms may be strengthened by inclusion of environmental factors in the analysis, which was not possible in our study due to lack of information on these variables. Due to a small sample size, we did not match cases of T2DM and controls by BMI to investigate whether or not obesity modulates the effect of polymorphisms on T2DM.

\section{Conclusion}

Our study has conducted a genetic association of the FTO, ENPP1, and TCF7L2 polymorphisms with T2DM and related traits. We found significant association between the rs997509 SNP while the rs7903146 and rs9941349 demonstrated borderline correlation with T2DM in the South African mixed ancestry ethnic population. A genome-wide association analysis in a large sample size is required to identify polymorphisms that may predispose mixed ancestry individuals to T2DM, accounting for ancestry genetic background.

\section{Acknowledgements}

We would like to express our gratitude to the Bellville South Community of Cape Town, South Africa. This research was supported by grants from the South African Medical Research Council, and University Research Fund of the Cape Peninsula University of Technology, South Africa.

\section{Disclosure statement}

None for all co-authors. 
Contribution statement: YYY: conception and design, acquisition and interpretation of data, preparation of the first draft and approval of final draft. JHM acquisition and interpretation of data, and approval of final draft. APK: analysis and interpretation of data, revision for important intellectual content and approval of final draft. RTE: acquisition of data, revision for important intellectual content and approval of final draft. TSP: revision for important intellectual content and approval of final draft. TEM: conception and design, acquisition and interpretation of data, revision for important intellectual content and approval of final draft.

\section{References}

1. Qi Q, Hu FB. Genetics of type 2 diabetes in European populations. J Diabetes. 2012;4:203-12. doi: 10.1111/j.1753-0407.2012.00224.x

2. Yu W, Hu C, Jia W. Genetic advances of type 2 diabetes in Chinese populations. J Diabetes. 2012;4:213-20 PubMed . doi: 10.1111/j.1753-0407.2012.00225.x

3. Grant SF, Thorleifsson G, Reynisdottir I, et al. Variant of transcription factor 7-like 2 (TCF7L2) gene confers risk of type 2 diabetes. Nat Genet. 2006;38 320- PubMed ;23. doi: 10.1074/jbc.M411487200. doi:10.1038/ ng1732

4. Yi F, Brubaker PL, Jin T. TCF-4 mediates cell type-specific regulation of proglucagon gene expression by beta-catenin and glycogen synthase kinase-3beta. J Biol Chem. 2005;280:1457-64 PubMed . doi: 10.1074/ jbc.M411487200

5. Damcott CM, Pollin TI, Reinhart LJ, et al. Polymorphisms in the transcription factor 7-like 2 (TCF7L2) gene are associated with type 2 diabetes in the Amish: replication and evidence for a role in both insulin secretion and insulin resistance. Diabetes. 2006;55:2654-9 PubMed . doi: 10.2337/db06-0338

6. Frayling TM, Timpson NJ, Weedon MN,et al. A common variant in the FTO gene is associated with body mass index and predisposes to childhood and adult obesity. Science. 2007;316:889- PubMed ;94. doi: 10.1126/ science. 1141634

7. Scuteri A, Sanna S, Chen WM, et al. Genome-Wide Association Scan Shows Genetic Variants in the FTO Gene Are Associated with Obesity-Related Traits. PLoS Genet. 2007;3:e115. doi: 10.1371/journal.pgen.0030115 8. Renström F, Payne F, Nordström A, et al. Replication and extension of genome-wide association study results for obesity in 4923 adults from northern Sweden. Hum
Mol Genet. 2009;18:1489-96 PubMed . doi: 10.1093/ $\mathrm{hmg} / \mathrm{ddp} 041$

9. Vasan SK, Karpe F, Gu HF, et al. FTO genetic variants and risk of obesity and type 2 diabetes: a meta-analysis of 28,394 Indians. Obesity (Silver Spring). 2014;22:964-70. doi: 10.1002/oby.20606

10. Hertel JK, Johansson S, Sonestedt E, et al. FTO, type 2 diabetes, and weight gain throughout adult life: a meta-analysis of 41,504 subjects from the Scandinavian HUNT, MDC, and MPP studies. Diabetes. 2011;60:163744 PubMed . doi; 10.2337/db10-1340.

11. Rees SD, Islam M, Hydrie MZ, et al. An FTO variant is associated with Type 2 diabetes in South Asian populations after accounting for body mass index and waist circumference. Diabet Med. 2011;28:673-80 PubMed . doi: 10.1111/j.1464-5491.2011.03257.x.

12. McAteer JB, Prudente S, Bacci S, et al. The ENPP1 $\mathrm{K} 121 \mathrm{Q}$ polymorphism is associated with type 2 diabetes in European populations: evidence from an updated meta-analysis I 42,042 subjects. Diabetes. 2008;57:112530 PubMed . doi: 10.2337/db07-1336

13. Seo HJ, Kim SG, Kwon OJ. The K121Q polymorphism in ENPP1 (PC-1) is not associated with type 2 diabetes or obesity in Korean male workers. J Korean Med Sci. 2008;23:459-64 PubMed . doi: 10.3346/ jkms.2008.23.3.459 PubMed

14. Li YY. ENPP1 K121Q polymorphism and type 2 diabetes mellitus in the Chinese population: a meta-analysis including 11,855 subjects. Metabolism. 2012;61:62533. doi: 10.1016/j.metabol.2011.10.002.

15. Goldfine ID, Maddux BA, Youngren JF, et al. The role of membrane glycoprotein plasma cell antigen 1/ ectonucleotide pyrophosphatase phosphodiesterase 1 in the pathogenesis of insulin resistance and related abnormalities. Endocr Rev. 2008;29:62-75. doi: 10.1210/ er.2007-0004

16. Ezzidi I, Mtiraoui N, Cauchi S, et al. Contribution of type 2 diabetes associated loci in the Arabic population from Tunisia: a case-control study. BMC Med Genet. 2009;10:33. PubMed doi: 10.1186/1471-2350-10-33.

17. Bouhaha R, Choquet H, Meyre D, et al. TCF7L2 is associated with type 2 diabetes in nonobese individuals from Tunisia. Pathol Biol (Paris). 2010;58:426-9. doi: 10.1016/j.patbio.2009.01.003

18. Pirie FJ, Motala AA, Pegoraro RJ, Paruk IM, Govender T, Rom L. Variants in PPARG, KCNJ11, TCF7L2, FTO, and HHEX genes in South African subjects of Zulu descent with type 2 diabetes. Afr J Diab Med. 2010;18:12-16. 
19. Cauchi S, Ezzidi I, El Achhab Y, et al. European genetic variants associated with type 2 diabetes in North African Arabs. Diabetes Metab. 2012;38:316-23 PubMed . doi: $10.1016 /$ j.diabet.2012.02.003

20. Danquah I, Othmer T, Frank LK, Bedu-Addo G, Schulze MB, Mockenhaupt FP. The TCF7L2 rs7903146 (T) allele is associated with type 2 diabetes in urban Ghana: a hospital-based case-control study. BMC Med Genet. 2013;14:96. doi: 10.1186/1471-2350-14-96

21.Vergotine Z, Yako YY, Kengne AP, Erasmus RT, Matsha TE. Proliferator-activated receptor gamma Pro$12 \mathrm{Ala}$ interacts with the insulin receptor substrate $1 \mathrm{Gl}-$ y972Arg and increase the risk of insulin resistance and diabetes in the mixed ancestry population from South Africa. BMC Genet. 2014;15:10. doi: 10.1186/14712156-15-10

22. Cho YS, Chen $\mathrm{CH}, \mathrm{Hu} \mathrm{C}$, et al. Meta-analysis of genome-wide association studies identifies eight new loci for type 2 diabetes in east Asians. Nat Genet. 2011;44:677223 PubMed . doi: 10.1038/ng.1019

23. Sakai K, Imamura M, Tanaka Y, et al.Replication study for the association of 9 East Asian GWAS-derived loci with susceptibility to type 2 diabetes in a Japanese population. PLoS One. 2013;8:e76317. doi: 10.1371/journal.pone.0076317

24. Vergotine Z, Yako YY, Kengne AP, Erasmus RT, Matsha TE. Insulin Receptor Substrate-1 Gly972Arg variant is not associated with type 2 diabetes in a South African population. S Afri Med J. 2014;104:420-423. doi: 10.7196/samj.7419

25. de Wit E, Delport W, Rugamika CE, et al. Genome-wide analysis of the structure of the South African coloured population in the Western Cape. Hum Genet. 2010;128:145-53 PubMed . doi: 10.1007/s00439010-0836-1

26. Zemlin AE, Matsha TE, Hassan MS, Erasmus RT. $\mathrm{HbA} 1 \mathrm{c}$ of $6.5 \%$ to diagnose diabetes mellitus - does it work for us? -the Bellville South Africa study. PloS One 2011; 6: e22558. doi: 10.1371/journal.pone.0022558

27. Matsha TE, Hassan MS, Kidd M, Erasmus RT. The 30-year cardiovascular risk profile of South Africans with diagnosed diabetes, undiagnosed diabetes, pre-diabetes or normoglycaemia: the Bellville, South Africa pilot study. Cardiovasc J Afr. 2012;23:5-11. doi: 10.5830/ CVJA-2010-087

28. Chalmers J, MacMahon S, Mancia G, et al. 1999 World Health Organization-International Society of Hypertension Guidelines for the management of hypertension. Guidelines sub-committee of the World Health Organization. Clin Exp Hypertens. 1999;21:100960 PubMed . doi: 10.3109/10641969909061028
29. Alberti KG, Zimmet PZ. Definition, diagnosis and classification of diabetes mellitus and its complications. Part 1: diagnosis and classification of diabetes mellitus provisional report of a WHO consultation. Diabet Med. 1998;15:539-53. PMID: 9686693

30. Sladek R, Rocheleau G, Rung J, et al. A genome-wide association study identifies novel risk loci for type 2 diabetes. Nature 2007;445:881- PubMed ;85. doi:10.1038/ nature 05616

31. Cauchi S, Nead KT, Choquet H, et al. The genetic susceptibility to type 2 diabetes may be modulated by obesity status: implications for association studies. BMC Med Genet. 2008;9:45. PubMed doi: 10.1186/14712350-9-45

32. Uma Jyothi K, Jayaraj M, Subburaj KS, et al. Association of TCF7L2 gene polymorphisms with T2DM in the population of Hyderabad, India. PLoS One. 2013;8:e60212. doi: 10.1371/journal.pone.0060212

33. Guo T, Hanson RL, Traurig M, et al. TCF7L2 is not a major susceptibility gene for type 2 diabetes in Pima Indians: analysis of 3,501 individuals. Diabetes. 2007;56:3082-8 PubMed . doi: 10.2337/db07-0621

34. Ren Q, Han XY, Wang F, et al. Exon sequencing and association analysis of polymorphisms in TCF7L2 with type 2 diabetes in a Chinese population. Diabetologia. 2008;51:1146- PubMed ;52. doi: 10.1007/s00125008-1039-3

35. Chang YC, Chiu YF, Ho LL, et al. TCF7L2 genetic variants and progression to diabetes in the Chinese population: pleiotropic effects on insulin secretion and insulin resistance. J Mol Med (Berl). 2010;88:183-92. doi: 10.1007/s00109-009-0542-4

36. Kifagi C, Makni K, Boudawara M, et al. Association of Genetic Variations in TCF7L2, SLC30A8, HHEX, LOC387761, and EXT2 with Type 2 Diabetes Mellitus in Tunisia. Genet Test Mol Biomarkers. 2011;15:399-405. doi: $10.1089 / \mathrm{gtmb} .2010 .0199$

37. Zheng X, Ren W, Zhang S, et al. Association of type 2 diabetes susceptibility genes (TCF7L2, SLC30A8, PCSK1 and PCSK2) and proinsulin conversion in a Chinese population. Mol Biol Rep 2012;39:17- PubMed ;23. doi: 10.1007/s11033-011-0705-6.

38. Park SE, Lee WY, Oh KW, et al. Impact of common type 2 diabetes risk gene variants on future type 2 diabetes in the nondiabetic population in Korea.J Hum Genet. 2012;57:265- PubMed ;68. doi: 10.1038/jhg.2012.16

39. Florez JC, Jablonski KA, Bayley N, et al.TCF7L2 polymorphisms and progression to diabetes in the Diabetes Prevention Program. N Engl J Med 2006;355:241PubMed ;50. doi: 10.1056/NEJMoa062418

40. Pearson ER, Donnelly LA, Kimber C, et al. Varia- 
tion in TCF7L2 influences therapeutic response to sulfonylureas: a GoDARTs study. Diabetes. 2007;56:217882. doi:10.2337/db07-0440

41. 't Hart LM, Fritsche A, Nijpels G,et al. The CTRB1/2 locus affects diabetes susceptibility and treatment via the incretin pathway. Diabetes. 2013; 62:3275-81 PubMed . doi: 10.2337/db13-0227

42. Fisher E, Boeing H, Fritsche A, Doering F, Joost HG, Schulze MB. Whole-grainconsumptionand transcriptionfactor-7-like 2 (TCF7L2) rs7903146: gene-diet interaction in modulating type 2 diabetes risk. Br J Nutr. 2009;101:478-81. doi: 10.1017/S0007114508020369

43. Cornelis MC, Qi L, Kraft P, Hu FB. TCF7L2, dietarycarbohydrate, and risk of type 2 diabetes in US women. Am J ClinNutr. 2009; 89:1256-62. doi: 10.3945/ ajcn.2008.27058

44.Hindy G, Sonestedt E, Ericson U, et al. Role of TCF7L2 risk variant and dietary fibre intake on incident type 2 diabetes. Diabetologia. 2012; 55:2646- PubMed ;2654. doi: 10.1007/s00125-012-2634-x

45. Bochenski J, Placha G, Wanic K, et al. New polymorphism of ENPP1 (PC-1) is associated with increased risk of type 2 diabetes among obese individuals. Diabetes 2006;55:2626- PubMed ;30. doi: 10.2337/db06-0191

46. Robiou-du-Pont S, Bonnefond A, Yengo L, et al.
Contribution of 24 obesity-associated genetic variants to insulin resistance, pancreatic beta-cell function and type 2 diabetes risk in the French population. Int J Obes (Lond). 2013; 37:980-5. doi: 10.1038/ijo.2012.175

47. Hassanein MT, Lyon HN, Nguyen TT, et al. Fine mapping of the association with obesity at the FTO locus in African-derived populations. HumMol Genet. 2010;19:2907-16. doi: 10.1093/hmg/ddq178

48. Moore AF, Jablonski KA, Mason CC, et al. The association of ENPP1 K121Q with diabetes incidence is abolished by lifestyle modification in the diabetes prevention program. J Clin Endocrinol Metab. 2009;94:44955. doi: $10.1210 /$ jc.2008-1583

49. Ortega-Azorín C, Sorlí JV, Asensio EM, et al. Associations of the FTO rs9939609 and the MC4R rs17782313 polymorphisms with type 2 diabetes are modulated by diet, being higher when adherence to the Mediterranean diet pattern is low. Cardiovasc Diabetol. 2012;11:137. doi: 10.1186/1475-2840-11-137

50. Mattei J,Qi Q, Hu FB, Sacks FM, Qi L. TCF7L2rs12255372-TT risk genotype and fat intake for changes in BMI, total fat mass, and trunk fat mass at 6 month of lifestyle intervention. Am J Clin Nutr. 2012;96:1129_ PubMed ;36. doi: 10.3945/ajcn.112.038125 